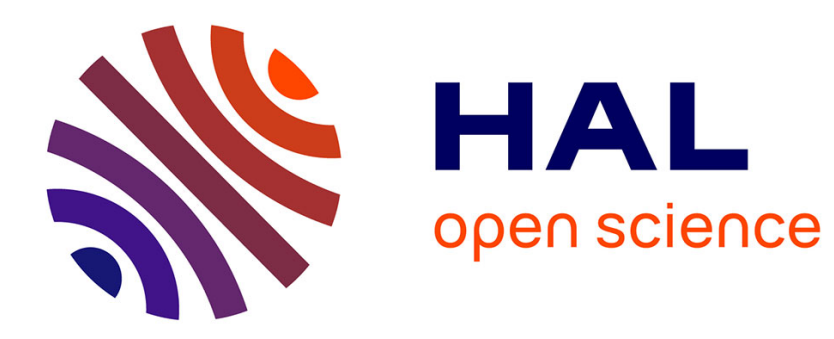

\title{
Multivariate shuffles and approximation of copulas
}

Fabrizio Durante, Juan Fernández-Sánchez

\section{To cite this version:}

Fabrizio Durante, Juan Fernández-Sánchez. Multivariate shuffles and approximation of copulas. Statistics and Probability Letters, 2010, 10.1016/j.spl.2010.08.008 . hal-00691784

\section{HAL Id: hal-00691784 \\ https://hal.science/hal-00691784}

Submitted on 27 Apr 2012

HAL is a multi-disciplinary open access archive for the deposit and dissemination of scientific research documents, whether they are published or not. The documents may come from teaching and research institutions in France or abroad, or from public or private research centers.
L'archive ouverte pluridisciplinaire HAL, est destinée au dépôt et à la diffusion de documents scientifiques de niveau recherche, publiés ou non, émanant des établissements d'enseignement et de recherche français ou étrangers, des laboratoires publics ou privés. 


\section{Accepted Manuscript}

Multivariate shuffles and approximation of copulas

Fabrizio Durante, Juan Fernández-Sánchez

PII:

S0167-7152(10)00231-2

DOI:

10.1016/j.spl.2010.08.008

Reference: STAPRO 5767

To appear in: Statistics and Probability Letters

Received date: 26 May 2010

Revised date: 11 August 2010

Accepted date: 12 August 2010

Please cite this article as: Durante, F., Fernández-Sánchez, J., Multivariate shuffles and approximation of copulas. Statistics and Probability Letters (2010), doi:10.1016/j.spl.2010.08.008

This is a PDF file of an unedited manuscript that has been accepted for publication. As a service to our customers we are providing this early version of the manuscript. The manuscript will undergo copyediting, typesetting, and review of the resulting proof before it is published in its final form. Please note that during the production process errors may be discovered which could affect the content, and all legal disclaimers that apply to the journal pertain. 


\title{
Multivariate shuffles and approximation of copulas
}

\author{
Fabrizio Durante ${ }^{\mathrm{a}, *}$, Juan Fernández-Sánchez ${ }^{\mathrm{b}}$ \\ ${ }^{a}$ School of Economics and Management \\ Free University of Bozen-Bolzano, Bolzano, Italy \\ ${ }^{b}$ Grupo de Investigación de Análisis Matemático \\ Universidad de Almería, La Cañada de San Urbano, Almería, Spain
}

\begin{abstract}
We present and study a method for constructing multivariate copulas, which includes both the shuffles of Min and the ordinal sums. Such a method has been used in order to show that suitable transformations of a given copula constitute a dense set in the class of all copulas with respect to the $L^{\infty}$ norm.
\end{abstract}

Keywords: Copula, $d$-fold stochastic measure, ordinal sum, shuffle of Min.

2010 MSC: 62H05, 60E05

\section{Introduction}

Let $\mathbb{I}$ be the unit interval $[0,1]$ and let $\mathbb{I}^{d}$ be the $d$-dimensional unit cube $(d \geq 2)$. A $d$-dimensional copula (copula, for brevity) is a distribution function on $\mathbb{I}^{d}$ whose univariate margins are uniformly distributed on $\mathbb{I}$. Copulas have been widely studied in Probability and Statistics due to the fact that they capture the scale-invariant dependence of continuous random vectors, as shown by Sklar's Theorem (Sklar, 1959). For more information about copulas and their applications, see, e.g., Jaworski et al. (2010); McNeil et al. (2005); Nelsen (2006); Salvadori et al. (2007).

A shuffle of Min (where Min indicates the comonotonicity copula $M_{d}\left(u_{1}, \ldots, u_{d}\right)=$ $\left.\min \left\{u_{1}, \ldots, u_{d}\right\}\right)$ is a construction principle that generates new copulas by means of a suitable rearrangement of the mass distribution of $M_{d}$ (Mikusiński et al., 1992, 2009). It has been used in several studies about approximations of copulas. In particular, it has been observed that shuffles of Min are dense in the class of bivariate copulas endowed with the $L^{\infty}$ norm (Mikusiński et al., 1992; Vitale, 1990).

Recently, Durante et al. (2009) have generalized the notion of shuffles of Min in order to allow that the probability mass of any bivariate copula, not only $M_{2}$, can be used as a starting point for further rearrangements. Such a generalization is based on the use

\footnotetext{
${ }^{*}$ Corresponding author. Tel. +39 0471013493. Fax +39 0471013009.

Email addresses: fabrizio.durante@unibz.it (Fabrizio Durante), juanfernandez@ual.es (Juan Fernández-Sánchez)
} 
of special measure-preserving transformations, which conveniently describe the geometric approach commonly used in defining shuffles of Min.

In this paper, we aim at providing a further construction principle that is directly inspired by the idea of shuffle of Min (see section 2). Such a method encompasses both the multivariate shuffle of Min presented by Mikusiński et al. (2009) and the ordinal sum construction by Mesiar and Sempi (2010). Remarkably, copulas obtained by the introduced method have an interesting stochastic representation.

Moreover, starting with any copula $C$, it has been shown that the set of all transformations of $C$ by means of such a construction is dense in the set of copulas endowed with the $L^{\infty}$ norm (see section 3 ). A feature of particular interest when one wants to approximate copulas by means of elements from a given basis set.

In order to reach our goal, we rely on measure-theoretic techniques that are grounded on the well-known one-to-one correspondence between copulas and special probability measures.

\section{Definitions and basic properties}

Let $d \in \mathbb{N}, d \geq 2$. Let $\lambda$ be the Lebesgue measure on $\mathbb{R}$. Let $\operatorname{Cop}_{d}$ be the set of all $d$-dimensional copulas, i.e. the set of all distribution functions $C: \mathbb{R}^{d} \rightarrow \mathbb{I}$ whose univariate margins are uniformly distributed on $\mathbb{I}$. Notice that, since a copula $C$ is uniquely determined by the values that it assumes on $\mathbb{I}^{d}$, we usually refrain from specifying the value of $C$ outside $\mathbb{I}^{d}$. For basic results and notations about copulas, we refer to Durante and Sempi (2010).

We recall that a copula $C$ induces a probability measure $\mu_{C}$ defined, for every nonempty orthotope $R \subseteq \mathbb{I}^{d}$, by $\mu_{C}(R):=V_{C}(R)$, where $V_{C}(R)$ denotes the $C$-volume of $R$, and extended by means of standard arguments to the Borel $\sigma$-algebra $\mathscr{B}\left(\mathbb{I}^{d}\right)$ (Nelsen, 2006). Such a $\mu_{C}$ is $d$-fold stochastic, i.e. the push-forward of $\mu_{C}$ under any projection $p_{i}$ coincides with Lebesgue measure on $\mathbb{I}, \mu_{C}\left(p_{i}^{-1}(A)\right)=\lambda(A)$ for any Borel set $A \subseteq \mathbb{I}$. In particular, 2fold stochastic measures (better known as doubly stochastic measures) represent an infinitedimensional generalization of doubly stochastic matrices and originated from an idea by Birkhoff (1948, Problem 111). Notably, such measures also appear in several problems connected with optimal transportation (Ahmad et al., 2009; Gangbo and McCann, 2000; Rachev and Rüschendorf, 1998).

Actually, $d$-fold stochastic measures on $\left(\mathbb{I}^{d}, \mathscr{B}\left(\mathbb{I}^{d}\right)\right)$ are in a one-to-one correspondence to the set of $d$-copula. In fact, from any $d$-fold stochastic measure $\mu$ a copula can be constructed by setting, for any $\mathbf{u} \in \mathbb{I}^{d}$,

$$
C_{\mu}(\mathbf{u}):=\mu([0, \mathbf{u}]),
$$

where $[0, \mathbf{u}]=\left[0, u_{1}\right] \times \cdots \times\left[0, u_{d}\right]$. In the following, when no confusion may arise, we may identify a copula and its corresponding measure.

Based on the isomorphism between copulas and measures, we propose the following construction principle for copulas. 
Definition 2.1. Let $\mathscr{J}^{1}, \ldots, \mathscr{J}^{d}$ be systems of closed and non-empty intervals of $\mathbb{I}, \mathscr{J}^{i}=$ $\left(J_{n}^{i}=\left[a_{n}^{i}, b_{n}^{i}\right]\right)_{n \in N}$ such that:

(S1) $N$ represents a finite or countable index set, i.e. $N=\{0,1, \ldots, \widetilde{n}\}$ or $N=\mathbb{Z}_{+}$;

(S2) for every $i \in\{1,2, \ldots, d\}$ and $n, m \in N, n \neq m, J_{n}^{i}$ and $J_{m}^{i}$ have at most one endpoint in common;

(S3) for every $i \in\{1,2, \ldots, d\}, \sum_{n \in N} \lambda\left(J_{n}^{i}\right)=1$;

(S4) for every $n \in N, \lambda\left(J_{n}^{1}\right)=\lambda\left(J_{n}^{2}\right)=\cdots=\lambda\left(J_{n}^{d}\right)$.

A system $S=\left(\mathscr{J}^{i}\right)_{i=1}^{d}$ satisfying the above properties is called shuffling structure (shortly, $S$-structure). The set of all S-structures based on an index set $N$ is indicated by $\mathscr{S}_{N}$, while $\mathscr{S}=\cup \mathscr{S}_{N}$.

Definition 2.2. Let $N$ be an index set as in $(\mathrm{S} 1)$. Let $\left(\mu_{n}\right)_{n \in N}$ be a system of probability measures on $\left(\mathbb{I}^{d}, \mathscr{B}\left(\mathbb{I}^{d}\right)\right)$ such that, for every $n \in N, \mu_{n}\left((0,1)^{d}\right)=1$. Let $\left(\mathscr{J}^{i}\right)_{i=1}^{d}$ be an S-structure. Let $\mu: \mathscr{B}\left(\mathbb{I}^{d}\right) \rightarrow \mathbb{R}_{+}$be the set-function defined, for every $A \in \mathscr{B}\left(\mathbb{I}^{d}\right)$, by

$$
\mu(A)=\sum_{\left(n_{1}, \ldots, n_{d}\right) \in N^{d}} \mu_{n_{1}, \ldots, n_{d}}\left(A \cap\left(J_{n_{1}} \times \cdots \times J_{n_{d}}\right)\right),
$$

where, for all $\left(n_{1}, \ldots, n_{d}\right) \in N^{d}, \mu_{n_{1}, \ldots, n_{d}}$ is the set-function defined on the Borel sets of $J_{n_{1}}^{1} \times \cdots \times J_{n_{d}}^{d}$ in the following way:

(M1) $\mu_{n_{1}, \ldots, n_{d}}=0$ if $n_{k} \neq n_{k^{\prime}}$ for some $k, k^{\prime}$;

(M2) for every Borel set $A \subseteq J_{n}^{1} \times \cdots \times J_{n}^{d}$

$$
\mu_{n, \ldots, n}(A)=\lambda\left(J_{n}^{1}\right) \mu_{n}\left(\varphi_{n}(A)\right),
$$

where $\varphi_{n}: J_{n}^{1} \times \cdots \times J_{n}^{d} \rightarrow \mathbb{I}^{d}$ is given by

$$
\varphi_{n}\left(x_{1}, \ldots, x_{d}\right)=\left(\frac{x_{1}-a_{n}^{1}}{\lambda\left(J_{n}^{1}\right)}, \ldots, \frac{x_{d}-a_{n}^{d}}{\lambda\left(J_{n}^{1}\right)}\right) .
$$

The set-function $\mu$ is called shuffling set-function related to the S-structure $\left(\mathscr{J}^{i}\right)_{i=1}^{d}$ and to $\left(\mu_{n}\right)_{n \in N}$. It is indicated by the symbol $\mu=\left\langle\left(\mathscr{J}^{i}\right)_{i=1}^{d},\left(\mu_{n}\right)_{n \in N},\right\rangle$.

Notice that, by definition, a shuffling set-function $\mu$ is a measure, since any $\mu_{n_{1}, \ldots, n_{d}}$ is a measure and $\cup_{\left(n_{1}, \ldots, n_{d}\right) \in N^{d}}\left(J_{n_{1}}^{1} \times \cdots \times J_{n_{d}}^{d}\right)=\mathbb{I}^{d}$. Moreover, the following result can be proved.

Proposition 2.1. Let $\mu=\left\langle\left(\mathscr{J}^{i}\right)_{i=1}^{d},\left(\mu_{n}\right)_{n \in N},\right\rangle$ be a shuffling set-function. Then $\mu$ is a probability measure. Moreover, if each $\mu_{n}$ is $d$-fold stochastic, then $\mu$ is d-fold stochastic. 
Proof. Let $\mu=\left\langle\left(\mathscr{J}^{i}\right)_{i=1}^{d},\left(\mu_{n}\right)_{n \in N},\right\rangle$ be a shuffling set-function. Then

$$
\begin{aligned}
\mu\left(\mathbb{I}^{d}\right) & =\sum_{\left(n_{1}, \ldots, n_{d}\right) \in N^{d}} \mu_{n_{1}, \ldots, n_{d}}\left(\cup_{\left(n_{1}, \ldots, n_{d}\right) \in N^{d}}\left(J_{n_{1}}^{1} \times \cdots \times J_{n_{d}}^{d}\right)\right) \\
& =\sum_{n \in N} \mu_{n, \ldots, n}\left(J_{n}^{1} \times \cdots \times J_{n}^{d}\right)=\sum_{n \in N} \lambda\left(J_{n}^{1}\right) \mu_{n}\left(\varphi_{n}\left(J_{n}^{1} \times \cdots \times J_{n}^{d}\right)\right)=1,
\end{aligned}
$$

that is $\mu$ is a probability measure.

Now, suppose that each $\mu_{n}$ is $d$-fold stochastic. In order to prove that $\mu$ is $d$-fold stochastic, we have to show that, for every every $i \in\{1, \ldots, d\}$ and for every $x_{i} \in \mathbb{I}$,

$$
\mu\left(\mathbb{I}^{i-1} \times\left[0, x_{i}\right] \times \mathbb{I}^{d-i}\right)=\lambda\left(\left[0, x_{i}\right]\right) .
$$

Suppose that $i=1$ (the other cases can be treated analogously). Let $x_{1} \in \mathbb{I}$. Since $\mu\left(\left\{x_{1}\right\} \times \mathbb{I}^{d-1}\right)=0$, we can assume, without loss of generality, that $x_{1} \notin\left\{a_{n}^{1}, b_{n}^{1}\right\}$ for every $n \in N$. Let $N_{1}$ be the following index set

$$
N_{1}=\left\{n \in N: J_{n}^{1} \cap\left[0, x_{1}\right] \neq \emptyset \text { and } x_{1} \notin J_{n}^{1}\right\} .
$$

Let $\widehat{n} \in N$ such that $x_{1} \in\left[a_{\widehat{n}}^{1}, b_{\widehat{n}}^{1}\right]$. It follows that

$$
\begin{aligned}
\mu\left(\left[0, x_{1}\right] \times \mathbb{I}^{d-1}\right)= & \left(\sum_{n \in N_{1}} \lambda\left(J_{n}^{1}\right) \mu_{n}\left(\varphi_{n}\left(J_{n}^{1} \times \cdots \times J_{n}^{d}\right)\right)\right) \\
& +\lambda\left(J_{\widehat{n}}^{1}\right) \mu_{\widehat{n}}\left(\varphi_{\widehat{n}}\left(\left(J_{\widehat{n}}^{1} \cap\left[0, x_{1}\right]\right) \times J_{\widehat{n}}^{2} \times \cdots \times J_{\widehat{n}}^{d}\right)\right) \\
= & \sum_{n \in N_{1}} \lambda\left(J_{n}^{1}\right)+\lambda\left(J_{\widehat{n}}^{1}\right) \mu_{\widehat{n}}\left(\left[0, \frac{x_{1}-a_{\widehat{n}}^{1}}{b_{\widehat{n}}^{1}-a_{\widehat{n}}^{1}}\right] \times \mathbb{I}^{d-1}\right) \\
= & \sum_{n \in N_{1}}\left(b_{n}^{1}-a_{n}^{1}\right)+\lambda\left(J_{\widehat{n}}^{1}\right) \frac{x_{1}-a_{\widehat{n}}^{1}}{b_{\widehat{n}}^{1}-a_{\widehat{n}}^{1}}=x_{1},
\end{aligned}
$$

which is the desired assertion.

A copula $C$ is a shuffling copula (S-copula, for short) if its induced measure $\mu_{C}$ can be represented as a shuffling measure that is $d$-fold stochastic. Such a $C$ is indicated by the symbol $\left\langle\left(\mathscr{J}^{i}\right)_{i=1}^{d},\left(C_{n}\right)_{n \in N}\right\rangle$, where $C_{n}$ is the copula such that $\mu_{n}=\mu_{C_{n}}$.

Remark 2.1. Intuitively, an S-copula $C$ is obtained by the following procedure:

1. define a suitable partition $\left\{J_{n_{1}}^{1} \times \cdots \times J_{n_{d}}^{d}\right\}_{\left(n_{1}, \ldots, n_{d}\right) \in N^{d}}$ of $\mathbb{I}^{d}$ formed by $d$-dimensional orthotopes (the S-structure);

2. given a system of copulas $\left(C_{n}\right)_{n \in N}$, plug a transformation of the probability mass of $C_{n}$ in $J_{n}^{1} \times \cdots \times J_{n}^{d}$,

3. define $C$ as the copula whose associated measure coincides in each $J_{n_{1}}^{1} \times \cdots \times J_{n_{d}}^{d}$ with the previous transformation of $\mu_{C_{n}}$. 
An explicit expression of an S-copula is given by the following result.

Proposition 2.2. Let $C=\left\langle\left(\mathscr{J}^{i}\right)_{i=1}^{d},\left(C_{n}\right)_{n \in N}\right\rangle$ be an $S$-copula. Then, for all $\mathbf{u} \in \mathbb{I}^{d}$,

$$
C(\mathbf{u})=\sum_{n \in N} \lambda\left(J_{n}^{1}\right) C_{n}\left(\frac{u_{1}-a_{n}^{1}}{\lambda\left(J_{n}^{1}\right)}, \ldots, \frac{u_{d}-a_{n}^{d}}{\lambda\left(J_{n}^{1}\right)}\right) .
$$

Proof. Since Definition 2.2 it follows that, for every $\mathbf{u} \in \mathbb{I}^{d}$,

$$
\begin{aligned}
C(\mathbf{u}) & =\mu_{C}([0, \mathbf{u}])=\sum_{n \in N} \lambda\left(J_{n}^{1}\right) \mu_{C_{n}}\left(\varphi_{n}\left([0, \mathbf{u}] \cap\left(J_{n}^{1} \times \cdots \times J_{n}^{d}\right)\right)\right) \\
& =\sum_{n \in N} \lambda\left(J_{n}^{1}\right) C_{n}\left(\frac{u_{1}-a_{n}^{1}}{\lambda\left(J_{n}^{1}\right)}, \ldots, \frac{u_{d}-a_{n}^{d}}{\lambda\left(J_{n}^{1}\right)}\right),
\end{aligned}
$$

which is the desired assertion.

Thanks to eq. (4), an S-copula can be interpreted as a convex combination (with coefficients $\left.\lambda\left(J_{n}^{1}\right)\right)$ of the $d$-variate distribution functions $F_{n}(\mathbf{x})=C_{n}\left(\frac{x_{1}-a_{n}^{1}}{\lambda\left(J_{n}^{1}\right)}, \ldots, \frac{x_{d}-a_{n}^{d}}{\lambda\left(J_{n}^{1}\right)}\right)$. As such, an S-copula $C=\left\langle\left(\mathscr{J}^{i}\right)_{i=1}^{d},\left(C_{n}\right)_{n \in N}\right\rangle$ has the following probabilistic interpretation, which is inspired by the methods of Mikusiński et al. (1991).

Let $(\Omega, \mathscr{A}, \mathbb{P})$ be a probability space. Assume that, for every $n \in N$, the random vector $\mathbf{U}^{n}=\left(U_{1}^{n}, \ldots, U_{d}^{n}\right)$ is distributed according to $C_{n}$. Let $Z$ be a discrete random variable assuming values in $N$ such that, for every $n \in N, P(Z=n)=\lambda\left(J_{n}^{1}\right)$. For every $n \in N$, consider the random vector

$$
\mathbf{V}^{n}=\left(V_{1}^{n}, \ldots, V_{d}^{n}\right)=\left(\lambda\left(J_{n}^{1}\right) U_{1}^{n}+a_{n}^{1}, \ldots, \lambda\left(J_{n}^{1}\right) U_{d}^{n}+a_{n}^{d}\right) .
$$

Finally, let us consider the random vector $\mathbf{W}$ given by

$$
\mathbf{W}=\sum_{n \in N} \sigma_{n}(Z) \mathbf{V}^{n}
$$

where, for every $n \in N, \sigma_{n}(x)=1$ if $x=n, \sigma_{n}(x)=0$ otherwise. For every $\mathbf{u} \in \mathbb{I}^{d}$

$$
\begin{aligned}
\mathbb{P}(\mathbf{W} \leq \mathbf{u}) & =\sum_{n \in N} \mathbb{P}(\mathbf{W} \leq \mathbf{u} \mid Z=n) \mathbb{P}(Z=n) \\
& =\sum_{n \in N} \lambda\left(J_{n}^{1}\right) \mathbb{P}\left(\mathbf{V}^{\mathbf{n}} \leq \mathbf{u}\right) \\
& =\sum_{n \in N} \lambda\left(J_{n}^{1}\right) C_{n}\left(\frac{u_{1}-a_{n}^{1}}{\lambda\left(J_{n}^{1}\right)}, \ldots, \frac{u_{d}-a_{n}^{d}}{\lambda\left(J_{n}^{1}\right)}\right),
\end{aligned}
$$

which coincides with (4).

Now, we show two relevant examples of S-copulas. 
Example 2.1. In the bivariate case, a copula $C$ is said to be shuffle of Min (Mikusiński et al., 1992, Definition 2.1) if there is a natural number $n$, two partitions

$$
0=s_{0}<s_{1}<\cdots<s_{\widetilde{n}}=1 \quad \text { and } \quad 0=t_{0}<t_{1}<\cdots<t_{\widetilde{n}}=1
$$

of $\mathbb{I}$, and a permutation $\varsigma$ of $\{1, \ldots, \widetilde{n}\}$ such that each $\left[s_{i-1}, s_{i}\right] \times\left[t_{\varsigma(i)-1}, t_{\varsigma(i)}\right]$ is a square in which $C$ distributes a mass $s_{i}-s_{i-1}$ uniformly spread along one of the diagonals.

Now, it can be easily proved that every shuffle of Min can be represented as an S-copula of the form $\left\langle\left(\mathscr{J}^{1}, \mathscr{J}^{2}\right),\left(C_{i}\right)_{i \in N}\right\rangle$, where:

- $N=\{0,1, \ldots, \widetilde{n}-1\}$

- $\mathscr{J}^{1}=\left(J_{i}^{1}\right)_{i \in N}$ is the partition given by $J_{i}^{1}=\left[s_{i-1}, s_{i}\right]$;

- $\mathscr{J}^{2}=\left(J_{i}^{2}\right)_{i \in N}$ is the partition given by $J_{i}^{2}=\left[t_{\varsigma(i-1)}, t_{\varsigma(i)}\right]$;

- $\left(C_{i}\right)_{i \in N}$ is a system of copulas where $C_{i}=M_{2}$, if the mass is distributed along the main diagonal of $\left[s_{i-1}, s_{i}\right] \times\left[t_{\varsigma(i)-1}, t_{\varsigma(i)}\right], C_{i}=W_{2}$, otherwise.

Recently, shuffles of Min have been also extended to the $d$-dimensional case (see (Mikusiński et al., 2009, section 6)). It is not difficult to show that also this extension can be represented in terms of S-copulas.

Example 2.2. Following Mesiar and Sempi (2010) (see also (Jaworski and Rychlik, 2008)), an ordinal sum of copulas can be introduced in the following way. Let $L$ be a finite or countable set, let $\left(\left[a_{k}, b_{k}\right]\right)_{k \in L}$ be a system of sub-intervals of $\mathbb{I}$, and let $\left(C_{k}\right)_{k \in L}$ be a system in $\operatorname{Cop}_{d}$. It is required that any two of the intervals $\left[a_{k}, b_{k}\right](k \in L)$ have at most one endpoint in common. Then the ordinal sum $C$ of $\left(C_{k}\right)_{k \in L}$ with respect to family of intervals $\left(\left[a_{k}, b_{k}\right]\right)_{k \in L}$ is the $d$-copula defined, for all $\mathbf{u} \in \mathbb{I}^{d}$ by

$$
C(\mathbf{u})=\left\{\begin{array}{c}
a_{k}+\left(b_{k}-a_{k}\right) C_{k}\left(\frac{\min \left\{u_{1}, b_{k}\right\}-a_{k}}{b_{k}-a_{k}}, \ldots, \frac{\min \left\{u_{d}, b_{k}\right\}-a_{k}}{b_{k}-a_{k}}\right), \\
\left.\quad \text { if } \min \left\{u_{1}, u_{2}, \ldots, u_{d}\right\} \in\right] a_{k}, b_{k}[\text { for some } k \in L, \\
\min \left\{u_{1}, u_{2}, \ldots, u_{d}\right\}, \quad \text { elsewhere. }
\end{array}\right.
$$

Now, such a $C$ can be represented as a suitable $\mathrm{S}$-copula. In order to show this, without loss of generality, suppose that $\left(\left[a_{k}, b_{k}\right]\right)_{k \in L}$ is a partition of $\mathbb{I}$. Then, $C$ given by $(5)$ is an S-copula of type $\left\langle\left(\mathscr{J}^{i}\right)_{i=1,2, \ldots, d},\left(C_{k}\right)_{k \in L}\right\rangle$, where, for $i=1, \ldots, d, \mathscr{J}^{i}=\left(J_{k}^{i}\right)_{k \in L}$ is the partition given by $J_{k}^{i}=\left[a_{k}, b_{k}\right]$.

Finally, we end the section with a characterization of S-copulas in terms of measurepreserving transformations. We recall that it is known from Vitale (1996) (see also de Amo et al. (2010); Kolesárová et al. (2008)) that every copula can be represented in the form

$$
C_{f_{1}, \ldots, f_{d}}(\mathbf{u})=\lambda\left(f_{1}^{-1}\left[0, u_{1}\right] \cap \cdots \cap f_{d}^{-1}\left[0, u_{d}\right]\right)
$$

for some measure-preserving transformations $f_{1}, \ldots, f_{d}$ of $\mathbb{I}$. 
Proposition 2.3. Let $C=\left\langle\left(\mathscr{J}^{i}\right)_{i=1}^{d},\left(C_{n}\right)_{n \in N}\right\rangle$ be an S-copula. Suppose that every $C_{n}$ can be represented in the form (6) by means of suitable $f_{1}^{n}, \ldots, f_{d}^{n}$. Then, $C$ can be represented in the form (6) where $f_{i}$, for $i=1,2, \ldots, d$, is given by

$$
f_{i}(t)= \begin{cases}f_{i}^{n}\left(\frac{t-a_{n}^{i}}{b_{n}^{i}-a_{n}^{i}}\right)\left(b_{n}^{i}-a_{n}^{i}\right)+a_{n}^{i}, & t \in] a_{n}^{i}, b_{n}^{i}[ \\ 0, & \text { otherwise. }\end{cases}
$$

Proof. Let $C=\left\langle\left(\mathscr{J}^{i}\right)_{i=1}^{d},\left(C_{n}\right)_{n \in N}\right\rangle$ be an S-copula. Let $C^{*}$ be the copula that can be represented in the form (6) where, for $i=1,2, \ldots, d, f_{i}$ is given by (7). In order to prove the assertion, it is enough to show that $\mu_{C}$ coincides with $\mu_{C^{*}}$ on every orthotope of type $J_{n}^{1} \times \cdots \times J_{n}^{d}$. Now, let $\mathbf{u} \in J_{n}^{1} \times \cdots \times J_{n}^{d}$. Then, it follows that:

$$
\begin{aligned}
& \mu_{C^{*}}\left(\left(a_{n}^{1}, u_{1}\right) \times \cdots \times\left(a_{n}^{d}, u_{d}\right)\right)=\lambda\left(\left\{t: f_{1}(t) \in\left(a_{n}^{1}, u_{1}\right), \ldots, f_{d}(t) \in\left(a_{n}^{d}, u_{d}\right)\right\}\right) \\
= & \left(b_{n}^{1}-a_{n}^{1}\right) \lambda\left(\left\{t: f_{n}^{1}(t) \in\left(0, \frac{u_{1}-a_{n}^{1}}{\lambda\left(J_{n}^{1}\right)}\right), \ldots, f_{n}^{d}(t) \in\left(0, \frac{u_{d}-a_{n}^{d}}{\lambda\left(J_{n}^{1}\right)}\right)\right\}\right) \\
= & \left(b_{n}-a_{n}\right) \mu_{C_{n}}\left(\left(0, \frac{u_{1}-a_{n}^{1}}{\lambda\left(J_{n}^{1}\right)}\right) \times \ldots \times\left(0, \frac{u_{d}-a_{n}^{d}}{\lambda\left(J_{n}^{1}\right)}\right)\right) \\
= & \mu_{C}\left(\left(a_{n}^{1}, u_{1}\right) \times \ldots \times\left(a_{n}^{d}, u_{d}\right)\right) .
\end{aligned}
$$

It follows that $\mu_{C^{*}}(A)=\mu_{C}(A)$ for every Borel set $A \subseteq J_{n}^{1} \times \cdots \times J_{n}^{d}$, which is the desired assertion.

\section{Approximation of copulas by means of shuffles}

Here we show how the construction principle that we have introduced can be used in order to provide a way for approximating (in the $L^{\infty}$ norm) any copula. Firstly, we will consider some general aspects of the shuffling transformation.

Let $N$ be an index set as in (S1). Let $\Psi: \mathscr{S}_{N} \times \operatorname{Cop}_{d}^{N} \rightarrow \operatorname{Cop}_{d}$ be the shuffling mapping defined by

$$
\left(\left(\mathscr{J}^{i}\right)_{i=1}^{d},\left(C_{n}\right)_{n \in N}\right) \stackrel{\Psi}{\longrightarrow}\left\langle\left(\mathscr{J}^{i}\right)_{i=1}^{d},\left(C_{n}\right)_{n \in N}\right\rangle
$$

which associates to each S-structure and to each system of copulas, the corresponding S-copula.

Now, for a fixed S-structure $S \in \mathscr{S}_{N}$, consider the function

$$
\Psi_{(S, \cdot)}: \operatorname{Cop}_{d}^{N} \rightarrow \operatorname{Cop}_{d}, \quad\left(C_{n}\right)_{n \in N} \mapsto\left\langle S,\left(C_{n}\right)_{n \in N}\right\rangle .
$$

Let $d_{\text {sup }}$ be the distance on $\operatorname{Cop}_{d}$ induced by the $L^{\infty}$ - norm. Let $d_{\text {sup }}^{N}$ be the distance defined on $\operatorname{Cop}_{d}^{N}$ by

$$
d_{\text {sup }}^{N}\left(\left(C_{n}\right)_{n \in N},\left(C_{n}^{\prime}\right)_{n \in N}\right)=\sup _{n \in N} \sup _{\mathbf{u} \in \mathbb{I}^{d}}\left|C_{n}(\mathbf{u})-C_{n}^{\prime}(\mathbf{u})\right|
$$

The following result holds. 
Proposition 3.1. For any $S \in \mathscr{S}_{N}, \Psi_{(S, \cdot)}$ is a Lipschitz mapping between $\left(\mathrm{Cop}_{d}, d_{\text {sup }}^{N}\right)$ and $\left(\mathrm{Cop}_{d}, d_{\text {sup }}\right)$.

Proof. Let $S \in \mathscr{S}_{N}$. Let $\left(C_{n}\right)_{n \in N}$ and $\left(C_{n}^{\prime}\right)_{n \in N}$ be in $\operatorname{Cop}_{d}^{N}$. Set $\Psi_{(S, \cdot)}\left(\left(C_{n}\right)_{n \in N}\right)=C$ and $\Psi_{(S, \cdot)}\left(\left(C_{n}^{\prime}\right)_{n \in N}\right)=C^{\prime}$. We have

$$
\begin{aligned}
\sup _{\mathbf{u} \in \mathbb{I}^{d}}\left|C(\mathbf{u})-C^{\prime}(\mathbf{u})\right| & =\sup _{n \in N}\left(\sup _{\mathbf{u} \in J_{n}^{1} \times \cdots \times J_{n}^{d}}\left|C(\mathbf{u})-C^{\prime}(\mathbf{u})\right|\right) \\
& \leq \sup _{n \in N} \lambda\left(J_{n}^{1}\right) \sup _{\mathbf{u} \in \mathbb{I}^{d}}\left|C_{n}(\mathbf{u})-C_{n}^{\prime}(\mathbf{u})\right| \\
& \leq\left(\sup _{n \in N} \lambda\left(J_{n}^{1}\right)\right) d_{\sup }^{N}\left(\left(C_{n}\right)_{n \in N},\left(C_{n}^{\prime}\right)_{n \in N}\right),
\end{aligned}
$$

which is the desired assertion.

Roughly speaking, for a given $S \in \mathscr{S}_{N}$, when two systems of copulas are close each other, so are the corresponding S-copulas.

Remark 3.1. Let $S \in \mathscr{S}_{N}$. Let $\left(C_{n}\right)_{n \in N}$ and $\left(C_{n}^{\prime}\right)_{n \in N}$ be in $\operatorname{Cop}_{d}^{N}$ such that, for every $\mathbf{u} \in \mathbb{I}^{d}, C_{n}(\mathbf{u}) \leq C_{n}^{\prime}(\mathbf{u})$ for every $n \in N$. Then it can be easily proved that $\Psi_{(S, \cdot)}\left(C_{n}\right)(\mathbf{u}) \leq$ $\Psi_{(S, \cdot)}\left(C_{n}^{\prime}\right)(\mathbf{u})$ for every $\mathbf{u} \in \mathbb{I}^{d}$. For more information about such a dependence order and its uses, see, for instance, (Joe, 1997; Nelsen, 2006).

A particular case of shuffling mapping is obtained when there exists $C \in \mathrm{Cop}_{d}$ such that $C_{n}=C$ for any $n \in N$. In such a case, for any $S \in \mathscr{S}_{N}$ the following mapping from $\mathrm{Cop}_{d}$ to $\mathrm{Cop}_{d}$ can be considered

$$
\widetilde{\Psi}_{(S, \cdot)}: \operatorname{Cop}_{d} \rightarrow \operatorname{Cop}_{d}, \quad C \mapsto\left\langle S,\left(C_{n}=C\right)_{n \in N}\right\rangle,
$$

A closer look at the proof of Proposition 3.1 allows to say that $\widetilde{\Psi}_{(S, \cdot)}$ is a contraction and, hence, it has a unique fixed point. A possible way for for determining the fixed point of $\widetilde{\Psi}_{(S, \cdot)}$ is described below.

Example 3.1. This example is based on some results about self-similar measures. For more details see (Hutchinson, 1981, section 4).

Let $\mathscr{X}$ be a complete metric space and let $\mathscr{P}$ be the family of all probability measures defined on the Borel $\sigma$-algebra associated with $\mathscr{X}$. For every (finite or countable) family $\left\{F_{t}\right\}_{t \in T}$ of contraction maps on $\mathscr{X}$ and every set of positive real numbers $\left\{\rho_{t}\right\}_{t \in T}$, we can define the mapping $F_{\rho}: \mathscr{P} \rightarrow \mathscr{P}$ such that $F_{\rho}(\mu)(A)=\sum_{t \in T} \rho_{t} \mu\left(F_{t}^{-1}(A)\right)$ for every Borel set $A$. It follows from (Hutchinson, 1981, subsection 4.4) that there exists a unique probability measure $\mu$ such that $F_{\rho}(\mu)=\mu$.

Now, let $S \in \mathscr{S}_{N}$. It can be easily proved that the fixed point of $\widetilde{\Psi}_{(S, \cdot)}$ is the copula $C$ such that its induced measure $\mu_{C}$ is associated with the family of contraction maps $\left\{F_{n}\right\}_{n \in N}$, where $F_{n}: \mathbb{I}^{d} \rightarrow J_{n}^{1} \times \cdots \times J_{n}^{d}$ is given by

$$
F_{n}\left(x_{1}, \ldots, x_{d}\right)=\lambda\left(J_{n}^{1}\right)\left(x_{1}, \ldots, x_{d}\right)+\left(a_{n}^{1}, \ldots, a_{n}^{d}\right),
$$


where, for $i \in\{1,2, \ldots, d\}, J_{n}^{i}=\left[a_{n}^{i}, b_{n}^{i}\right]$ and the set of positive real numbers is given by $\left\{\lambda\left(J_{n}^{1}\right)\right\}_{n \in N}$.

Now, given a copula $C$, let us consider the set $\left\{\widetilde{\Psi}_{(S, \cdot)}(C) \mid S \in \mathscr{S}\right\}$ formed by all $S-$ copulas generated from $C$ and any S-structure $S$ by means of $\widetilde{\Psi}_{(S, \cdot)}$. The following result holds.

Proposition 3.2. Let $C \in \operatorname{Cop}_{d}$. Then the set $\left\{\widetilde{\Psi}_{(S, \cdot)}(C) \mid S \in \mathscr{S}\right\}$ is dense in $\operatorname{Cop}_{d}$ with respect to the $L^{\infty}$ norm.

Proof. Let $C \in \operatorname{Cop}_{d}$. Let $p \in \mathbb{N}, p \geq 2$. Divide $\mathbb{I}^{d}$ into $p^{d}$ orthotopes of type

$$
B_{m}^{p}=\left[\frac{a_{m}^{1}}{p}, \frac{a_{m}^{1}+1}{p}\right] \times \cdots \times\left[\frac{a_{m}^{d}}{p}, \frac{a_{m}^{d}+1}{p}\right]
$$

where $m \in \mathscr{M}=\left\{0,1, \ldots, p^{d}-1\right\}, a_{m}^{i} \in\{0,1, \ldots, p-1\}$ for every $i \in\{1, \ldots, d\}$, and $m$ can be written in the form

$$
m=a_{m}^{1}+a_{m}^{2} p+\cdots+a_{m}^{d} p^{d-1} .
$$

We denote by $\mathscr{M}_{k, i}$ the subset of $\mathscr{M}$ formed by all $m \in \mathscr{M}$ such that $m$ can be written in the form (13) with $a_{m}^{i}=k$.

Notice that, since $\mu_{C}$ is $d$-fold stochastic, for every $m \in \mathscr{M}_{k, i}$

$$
\sum_{m \in \mathscr{M}_{k, i}} \mu_{C}\left(B_{m}^{p}\right)=\lambda\left(\mathbb{I}^{i-1} \times\left[\frac{k}{p}, \frac{k+1}{p}\right] \times \mathbb{I}^{d-i}\right)=\frac{1}{p} .
$$

Let $i \in\{1,2, \ldots, d\}$. Along the $i$-th axis, divide each interval of type $\left[\frac{k}{p}, \frac{k+1}{p}\right]$ into a system of closed intervals $\left(J_{m}^{i}\right)_{m \in \mathscr{M}_{k, i}}$ such that:

(a) $\lambda\left(J_{m}^{i}\right)=\mu_{C}\left(B_{m}^{p}\right)$ for every $m \in \mathscr{M}_{k, i}$;

(b) if $a_{m_{1}}^{i}<a_{m_{2}}^{i}$, then $\sup \left(J_{m_{1}}^{i}\right) \leq \inf \left(J_{m_{2}}^{i}\right)$;

(c) if $a_{m_{1}}^{i}=a_{m_{2}}^{i}$ and $m_{1}<m_{2}$, then $\sup \left(J_{m_{1}}^{i}\right) \leq \inf \left(J_{m_{2}}^{i}\right)$.

Notice that, because of condition (a), some of the intervals $J_{m}^{i}$ may be of measure zero. In such a case, they will not be considered.

By means of previous procedure, $\mathbb{I}$ can be partitioned into intervals in the following way:

$$
\mathbb{I}=\cup_{k=0}^{p-1}\left[\frac{k}{p}, \frac{k+1}{p}\right]=\cup_{k=0}^{p-1}\left(\cup_{m \in \mathscr{M}_{k, i}} J_{m}^{i}\right) .
$$

Let $\mathscr{J}^{i}=\left(J_{m}^{i}\right)_{m \in \mathscr{M}}$ be the system of intervals of eq. (14). Then it is easily proved that $\left(\mathscr{J}^{i}\right)_{i=1}^{d}$ forms an S-structure (which depends on $p$ ). Let $C_{p}$ be the S-copula of type 
$\left\langle\left(\mathscr{J}^{i}\right)_{i=1}^{d},\left(C_{m}=C\right)_{m \in \mathscr{M}}\right\rangle$. For every $B_{m}^{p}$ of type (12), it can be proved that $\mu_{C_{p}}\left(B_{m}^{p}\right)=$ $\mu_{C}\left(B_{m}^{p}\right)$.

Now, consider a continuous function $f$ on $\mathbb{I}^{d}$. Then, for every $\varepsilon>0$ and for every $m \in$ $\mathscr{M}$, there exists $q \in \mathbb{N}$ such that, for every $p>q$ and for every $\mathbf{x}, \mathbf{x}^{\prime} \in B_{m}^{p},\left|f(\mathbf{x})-f\left(\mathbf{x}^{\prime}\right)\right|<\varepsilon$. Therefore

$$
\begin{aligned}
\sum_{m} \min _{\mathbf{x} \in B_{m}^{p}}(f(\mathbf{x})) \mu_{C}\left(B_{m}^{p}\right) & =\sum_{m} \min _{\mathbf{x} \in B_{m}^{p}}(f(\mathbf{x})) \mu_{C_{p}}\left(B_{m}^{p}\right) \\
& \leq \int_{\mathbb{I}^{d}} f(\mathbf{x}) d \mu_{C_{p}} \leq \sum_{m} \max _{\mathbf{x} \in B_{m}^{p}}(f(\mathbf{x})) \mu_{C_{p}}\left(B_{m}^{p}\right) \\
& \leq \sum_{m} \max _{\mathbf{x} \in B_{m}^{p}}(f(\mathbf{x})) \mu_{C}\left(B_{m}^{p}\right) \leq \sum_{m} \min _{\mathbf{x} \in B_{m}^{p}}(f(\mathbf{x})) \mu_{C}\left(B_{m}^{p}\right)+\varepsilon,
\end{aligned}
$$

where the sums are taken over all possible indices $m$. It follows that

$$
\left|\int_{\mathbb{I}^{d}} f(\mathbf{x}) d \mu_{C_{p}}-\int_{\mathbb{I}^{d}} f(\mathbf{x}) d \mu_{C}\right|<\varepsilon
$$

when $p>q$. For the arbitrariness of $f$, we can conclude that $C_{p}$ converges pointwise to $C$ as $p$ tends to $+\infty$.

Remark 3.2. By using the same idea of the proof of Proposition 3.2, it should be noticed that, any $C \in \mathrm{Cop}_{d}$ could be approximated by means of S-copulas constructed from systems of copulas formed by elements not necessarily coinciding.

In the bivariate case, Proposition 3.2 was already known for the case $C=M_{2}$ (see (Mikusiński et al., 1992, Theorem 3.1)). In particular, since Proposition 3.2, it follows that the independence copula $\Pi_{d}(\mathbf{u})=u_{1} \cdots u_{d}$ can be approximated by means of elements of the set $\left\{\widetilde{\Psi}_{(S, \cdot)}\left(M_{d}\right) \mid S \in \mathscr{S}\right\}$. Intuitively speaking, taking into account the probabilistic interpretation of S-copulas and the fact that $M_{d}$ describe comonotone dependence, it follows that any sequence of independent random variables can be approximated by a sequence of random variables that are completely dependent (in the sense that each random variable is a deterministic function of the others). This intriguing fact was noted for the first time by Kimeldorf and Sampson (1978).

However, we want to stress that Proposition 3.2 strongly depends on the topology that we are considering over $\operatorname{Cop}_{d}$.

Consider, for example, the set of all finite signed measures on $\left(\mathbb{I}^{d}, \mathscr{B}\left(\mathbb{I}^{d}\right)\right)$. Such a set is a real vector space that, equipped with the total variation norm, forms a Banach space. It is known that a sequence of finite signed measure $\left(\mu_{n}\right)_{n}$ converges to $\mu$ if, and only if, for every $B \in \mathscr{B}\left(\mathbb{I}^{d}\right), \mu_{n}(B) \rightarrow \mu(B)$ as $n \rightarrow+\infty$.

Now, we can consider $\operatorname{Cop}_{d}$ (precisely, its induced measure) as subset of the set of all signed measures. Then $\mathrm{Cop}_{d}$ can be equipped with the topology induced by the total variation norm. In such a topology, it holds that, given $C \in \operatorname{Cop}_{d}$, the set $\left\{\widetilde{\Psi}_{(S, \cdot)}(C) \mid\right.$ $S \in \mathscr{S}\}$ need not be dense in $\operatorname{Cop}_{d}$. In fact, consider $C=M_{2}$. Then the elements 
of $\left\{\widetilde{\Psi}_{(S, \cdot)}\left(M_{2}\right) \mid S \in \mathscr{S}\right\}$ concentrates the probability mass on a set of 2 -dimensional Lebesgue measure 0 and, thus, they cannot approximate in the total variation norm the copula $\Pi_{2}$ (or, in general, any copula with a non-trivial absolutely continuous component).

\section{Acknowledgements}

The authors express their gratitude to the Referees for careful reading and some useful comments. The first author acknowledges the support of School of Economics and Management, Free University of Bozen-Bolzano, via the project "Multivariate dependence models".

\section{References}

Ahmad, N., Kim, H. K., McCann, R. J., 2009. Optimal transportation, topology and uniqueness, working paper.

Birkhoff, G., 1948. Lattice Theory. American Mathematical Society Colloquium Publications, vol. 25, revised edition. American Mathematical Society, New York, N. Y.

de Amo, E., Díaz Carrillo, M., Fernández Sánchez, J., 2010. Measure-preserving functions and the independence copula. Mediterr. J. Math., in press.

Durante, F., Sarkoci, P., Sempi, C., 2009. Shuffles of copulas. J. Math. Anal. Appl. 352 (2), 914-921.

Durante, F., Sempi, C., 2010. Copula theory: an introduction. In: Jaworki, P., Durante, F., Härdle, W., Rychlik, T. (Eds.), Workshop on Copula Theory and its Applications. Lecture Notes in Statistics Proceedings, pages 3-31. Springer, Berlin Heidelberg, 2010.

Gangbo, W., McCann, R. J., 2000. Shape recognition via Wasserstein distance. Quart. Appl. Math. 58 (4), 705-737.

Hutchinson, J. E., 1981. Fractals and self-similarity. Indiana Univ. Math. J. 30 (5), 713-747.

Jaworski, P., Durante, F., Härdle, W., Rychlik, T. (Eds.), 2010. Copula Theory and its Applications. Lecture Notes in Statistics - Proceedings. Springer, Berlin Heidelberg, 2010.

Jaworski, P., Rychlik, T., 2008. On distributions of order statistics for absolutely continuous copulas with applications to reliability. Kybernetika (Prague) 44 (6), 757-776.

Joe, H., 1997. Multivariate models and dependence concepts. Vol. 73 of Monographs on Statistics and Applied Probability. Chapman \& Hall, London.

Kimeldorf, G., Sampson, A. R., 1978. Monotone dependence. Ann. Statist. 6 (4), 895-903.

Kolesárová, A., Mesiar, R., Sempi, C., 2008. Measure-preserving transformations, copulæ and compatibility. Mediterr. J. Math. 5 (3), 325-339.

McNeil, A. J., Frey, R., Embrechts, P., 2005. Quantitative risk management. Concepts, techniques and tools. Princeton Series in Finance. Princeton University Press, Princeton, NJ.

Mesiar, R., Sempi, C., 2010. Ordinal sums and idempotents of copulas. Aequationes Math. 79 (1-2), 39-52. 
Mikusiński, P., , Taylor, M. D., 2009. Some approximations of n-copulas. Metrika, in press.

Mikusiński, P., Sherwood, H., Taylor, M. D., 1991. Probabilistic interpretations of copulas and their convex sums. In: Advances in probability distributions with given marginals (Rome, 1990). Vol. 67 of Math. Appl. Kluwer Acad. Publ., Dordrecht, pp. 95-112.

Mikusiński, P., Sherwood, H., Taylor, M. D., 1992. Shuffles of Min. Stochastica 13 (1), 61-74.

Nelsen, R. B., 2006. An introduction to copulas, 2nd Edition. Springer Series in Statistics. Springer, New York.

Rachev, S. T., Rüschendorf, L., 1998. Mass transportation problems. Vol. I-II. Probability and its Applications (New York). Springer-Verlag, New York.

Salvadori, G., De Michele, C., Kottegoda, N. T., Rosso, R., 2007. Extremes in Nature. An Approach Using Copulas. Vol. 56 of Water Science and Technology Library. Springer, Dordrecht (NL).

Sklar, A., 1959. Fonctions de répartition à $n$ dimensions et leurs marges. Publ. Inst. Statist. Univ. Paris $8,229-231$.

Vitale, R. A., 1990. On stochastic dependence and a class of degenerate distributions. In: Topics in statistical dependence (Somerset, PA, 1987). Vol. 16 of IMS Lecture Notes Monogr. Ser. Inst. Math. Statist., Hayward, CA, pp. 459-469.

Vitale, R. A., 1996. Parametrizing doubly stochastic measures. In: Rüschendorf, L., Schweizer, B., Taylor, M. (Eds.), Proceedings of the AMS-IMS-SIAM Joint Summer Research Conference on Distributions with Fixed Marginals, Doubly Stochastic Measures and Markov Operators held in Seattle, WA, August 1-5, 1993. Vol. 28 of IMS Lecture Notes Monogr. Ser. Inst. Math. Statist., Hayward, CA, pp. 358-364. 